\title{
Die volkswirtschaftliche Bedeutung der betriebswirtschaftlichen Rechnungslegung
}

\author{
Michael Drewes
}

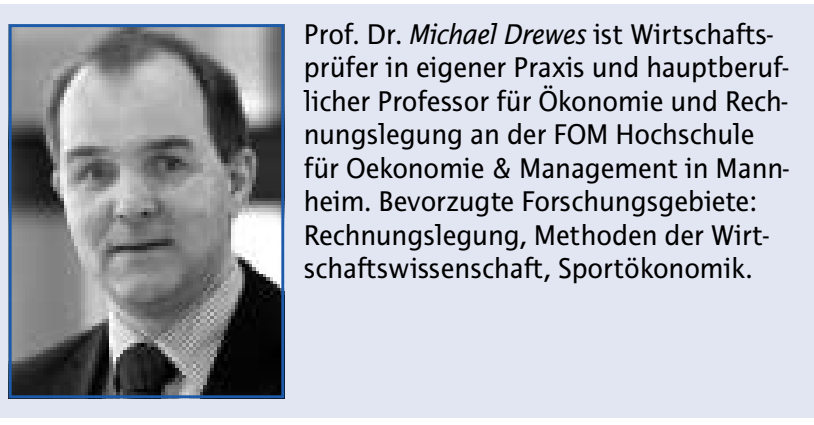

Der Aufsatz untersucht, welche volkswirtschaftlichen Auswirkungen die betriebswirtschaftliche Rechnungslegung hat. Dabei wird zum einen aus Sicht der Wohlfahrtsökonomie die Behebung von Marktversagenstatbeständen untersucht. Zum anderen wird die Bedeutung der Rechnungslegung für den Unternehmer beleuchtet, was vor allem durch die Erkenntnisse der Österreichischen Schule der Nationalökonomie verdeutlicht wird. Für die Fortentwicklung der Rechnungslegung bedeutet das, dass neben der Behebung von Informationsasymmetrien auch die notwendige Selbstinformation des Unternehmers berücksichtigt werden muss.

Stichwörter: Rechnungslegung, asymmetrische Informationsverteilung, Österreichische Schule, Handelsgesetzbuch, International Financial Reporting Standards

\section{Einleitung}

In Lehrbüchern zur Buchführung und zur Jahresabschlusserstellung werden als Zwecke von Buchführung und Jahresabschluss die Informationsfunktion und die Zahlungsbemessungsfunktion genannt. Als zentrale Aufgaben des Rechnungswesens und der Buchhaltung werden die Dokumentation (zur Rechenschaft), Information (insbesondere externer Adressaten), Steuerung, Kontrolle (Wirtschaftlichkeit und Liquidität) und Disposition (Planungsgrundlage) genannt. Die Buchhaltung dient als grundlegendes Instrument für das Controlling eines Unternehmens und spielt bei der Kontrolle der Geschäftsleitung eine Rolle (Littkemann/Holtrup/Schulte 2010, S. 4). Damit werden vornehmlich die betriebswirtschaftlichen Aufgaben der Rechnungslegung adressiert. Die betriebswirtschaftliche Rechnungslegung hat aber durchaus auch eine volkswirtschaftliche Bedeutung, da sie für das Funktionieren von Märkten unerlässlich ist. Dies wird vornehmlich in Form des Abbaus von asymmetrischen Informationsverteilun- gen oder anderer Marktversagenstatbestände in der Literatur erwähnt und diskutiert (z. B. Zimmermann 2002), so dass die Diskussion insbesondere auf wohlfahrtsökonomischen Überlegungen basiert. Diese wohlfahrtsökonomischen Erklärungsansätze sollen im zweiten Kapitel skizziert werden. Im dritten Kapitel wird jedoch eine weitere Rolle der betriebswirtschaftlichen Rechnungslegung für die Gesamtwirtschaft dargestellt, die sich aus Sicht der österreichischen Schule der Nationalökonomie ergibt, nämlich die Informationsverarbeitung und Orientierung im Markt für Unternehmen und Unternehmer. Im vierten Kapitel sollen Auswirkungen für die Weiterentwicklung der in Deutschland verwendeten Rechnungslegungssysteme (HGB und IFRS) diskutiert werden. Das fünfte Kapitel fasst die Überlegungen zusammen.

\section{Wohlfahrtsökonomische Erklärungsansätze}

Die neoklassisch geprägte Wohlfahrtsökonomie geht davon aus, dass es zu Fehlallokationen und Wohlfahrtsverlusten kommt, wenn Fälle des Marktversagens vorliegen. Bei Vorliegen von Marktversagen sind Lösungen zu suchen, die sich entweder aus wirtschaftspolitischer Aktivität oder aus Maßnahmen des privaten Sektors ergeben. Folgende Tatbestände können zu Marktversagen führen: Externalitäten, Marktmacht und Informationsasymmetrien. Für die Rechnungslegung spielt insbesondere die asymmetrische Verteilung von Informationen eine bedeutende Rolle. Von asymmetrischer Informationsverteilung wird gesprochen, wenn Anbieter und Nachfrager auf einem Markt auf unterschiedlichem Niveau informiert sind. Sie treten vor allem in sogenannten Agenturbeziehungen auf, bei denen der Prinzipal als Auftraggeber die besser und der Agent als Auftragnehmer die schlechter informierte Seite ist.

Küting/Lam (2013, S. 1737) sehen in dem gezielten Abbau von Informationsasymmetrien zwischen dem Rechnungslegenden und dem Rechnungslegungsadressaten den „grundlegenden Zweck einer jeden Rechnungslegung“. Externe Rechnungslegung dient insofern dem Abbau asymmetrischer Informationsverteilung, als mit ihrer Hilfe die besser informierte Geschäftsleitung (als Agent) den unterschiedlichen Interessengruppen eines Unternehmens (als Prinzipale) Informationen über die Vermögens-, Finanz- und Ertragslage zur Verfügung stellt bzw. stellen muss (Signalling). Die Interessengruppen können auf diese Weise ihren Informationsrückstand abbauen. So können beispielsweise kreditgebende Banken besser abschätzen, ob sie weitere Kredite an ein Unternehmen vergeben sol- 
len. Aktuelle und potenzielle Eigenkapitalgeber können Risiken und Chancen eines Investments sowie die Leistung der Geschäftsleitung (bei Trennung von Eigentum und Verfügungsmacht) besser beurteilen. Arbeitnehmer und Lieferanten erhalten Informationen über die Solidität des Unternehmens.

Im Extremfall können asymmetrische Informationsverteilungen zum Marktzusammenbruch führen (Akerlof 1970, 490). Aus diesem Grunde wird die Rechnungslegung reguliert bzw. durch private Maßnahmen geprägt, um auf diese Weise Informationsasymmetrien abzubauen und zum Funktionieren von Märkten beizutragen: Zum einen werden Standards erlassen, welche die Interpretation der Informationen erleichtern. Sie können die Form gesetzlicher Regelung annehmen (z. B. das Dritte Buch des Handelsgesetzbuches - HGB), als private Standards auftreten (z. B. International Accounting Standards - IAS oder International Financial Reporting Standards - IFRS) oder als allgemein anerkannte Vorgehensweise (z. B. Grundsätze ordnungsgemäßer Buchführung - GoB oder Generally Accepted Accounting Principles - GAAP) in Erscheinung treten. Zum anderen soll die Glaubwürdigkeit der Rechnungslegung durch externe Kontrolleure (d. h. insbesondere Abschlussprüfer) erhöht werden (Monitoring). Darüber hinaus können Marktteilnehmer die asymmetrische Informationsverteilung durch freiwillige Publizität abbauen, um Transaktionskosten zu senken oder Signale an die Marktgegenseite zu senden (Zimmermann 2002, S. 308).

Ist der Abbau von Informationsasymmetrien nach weit verbreiteter Ansicht der Hauptzweck einer jeden Rechnungslegung, zeigen sich jedoch Unterschiede in der Schwerpunktsetzung der einzelnen Rechnungslegungssysteme. In der internationalen Rechnungslegung, z. B. den US-GAAP oder den IFRS, steht die Informationsfunktion als alleiniger Rechnungslegungszweck im Vordergrund. Damit hat die Vermittlung entscheidungsnützlicher Informationen (,decision usefulness“") durch Abschlüsse nach den IFRS überragende Bedeutung; insofern stellen sie eine Reaktion auf die Informationsasymmetrien zwischen Abschlussersteller und Abschlussadressaten dar. Ziel der IFRS ist es demnach, die schlechter informierten Abschlussadressaten auf ein mit dem der besser informierten Abschlussersteller vergleichbares Informationsniveau zu heben. Dies soll erreicht werden, indem die Daten der externen Rechnungslegung auf den Informationen aus der internen Rechnungslegung basieren.

Die deutsche handelsrechtliche Rechnungslegung nach dem HGB verfolgt neben dem Zweck der Informationsvermittlung, mit dem asymmetrischen Informationsverteilungen Rechnungen getragen wird, zwei weitere Ziele. Zum einen ist das die Zahlungsbemessungsfunktion, mit der der ausschüttungsfähige Gewinn ermittelt wird, und die insofern dem Gläubigerschutzgedanken Rechnung trägt. Zum anderen wird mit der handelsrechtlichen Rechnungslegung auch eine Dokumentationsfunktion verfolgt, welche auf den GoB aufbaut. Diese Ziele stehen mindestens gleichberechtigt neben dem Zweck der Informationsver- mittlung an externe Stakeholder im Rahmen einer Agenturbeziehung.

\section{Rechnungslegung aus Österreichischer Sicht}

Mises (1922, S. 110) nannte die Buchführung das ,,vornehmste Mittel der ausgebildeten Rationalisierung des Handelns" und die Heimat der Wirtschaftsrechnung. Die Rolle ungleich verteilter Wissensstände für Kontrolle und Leitung von Unternehmen durch die Rechnungslegung hat die österreichische Schule der Nationalökonomie in ihrer Auseinandersetzung mit dem Sozialismus erkannt, ohne dies explizit zu formulieren (z. B. Hayek 1940, 140). Die Bedeutung der Rechnungslegung geht aber aus österreichischer Sicht über die Behebung von asymmetrischer Informationsverteilung hinaus. Um diese Bedeutung der Rechnungslegung aus Sicht der Österreichischen Schule der Nationalökonomie herauszuarbeiten, muss weiter ausgeholt werden: Zentrale Annahme der Österreichischen Schule der Nationalökonomie ist die ,unvermeidliche Unwissenheit der handelnden Menschen“ (Hayek 1996, 310), die auch als ,konstitutionelle Unwissenheit“ (z. B. Daumann/Drewes 2007, S. 81) bezeichnet wird. Der Markt wird als eine spontane Ordnung oder als ein dynamischer Prozess gesehen, der nur ausnahmsweise in einem Gleichgewichtszustand ist. Zentrale Aufgabe dieses dynamischen Prozesses ist die Schaffung und Verarbeitung von Wissen, das dezentral bei den Wirtschaftssubjekten entsteht bzw. vorhanden ist. Der Markt bündelt und koordiniert so eine Menge von Wissen, die Einzelpersonen oder eine Gruppe von Personen nie haben könnten.

Die unwissenden, kognitiv beschränkten Teilnehmer am dynamischen Marktprozess brauchen Informationen, welche ihnen Orientierung in einer komplexen, sich permanent ändernden und subjektiv wahrgenommenen Welt geben, um sinnvolle Entscheidungen treffen zu können. Sie erhalten diese Informationen über das Preissystem, welches ihnen die relativen Knappheiten anzeigt und aus dem Marktprozess resultiert (Huerta de Soto 2013, S. 118). Das Preissystem wirkt als abstrakte Datensammelstelle für das dezentral vorhandene Wissen, welche Informationen über relative Knappheit von Kapital, Gütern und Dienstleistungen gibt. Voraussetzung für das Funktionieren des Marktprozesses ist das Vorhandensein von Privateigentum an den Produktionsgütern. Ohne Privateigentum an den Produktionsfaktoren gibt es aus Sicht der österreichischen Schule der Nationalökonomie keinen Markt, und ohne Markt keine Marktpreise für die Produktionsfaktoren. Ohne Marktpreise fehlt den Marktteilnehmern wiederum die notwendige Information über die relativen Knappheiten sowie die Austauschverhältnisse zwischen verschiedenen Gütern und Produktionsfaktoren. Und ohne die notwendigen Informationen können keine sinnvollen Entscheidungen über die vielfältigen alternativen Einsatzmöglichkeiten des Kapitals getroffen werden (Boettke 1998, S. 134). Sinnvolle Entscheidungen sind im marktwirtschaftlichen Prozess überlebensnotwendig insbesondere für die Unter- 
nehmen, da sie dem marktwirtschaftlichen Sanktionsmechanismus unterworfen sind: Treffen die Unternehmen überwiegend sinnvolle Entscheidungen auf Basis der ausgewerteten Informationen, werden sie durch Gewinne belohnt und können am Markt verbleiben. Sind die Entscheidungen weniger sinnvoll, erleiden die Unternehmen Verluste und müssen bei anhaltenden Verlustsituationen aus dem Markt ausscheiden.

Die betriebswirtschaftliche Rechnungslegung entfaltet dabei Wirkung in zwei Richtungen: Die (externe) Rechnungslegung hilft den Unternehmen, retrospektiv auszuwerten, ob die in den Marktpreisen enthaltenen Informationen über relative Knappheiten richtig interpretiert wurden. In der deutschen Gesetzgebung wurde ursprünglich der Zweck der Buchführung in der ,,persönlichen Rechenschaftslegung des Kaufmanns im Interesse einer erfolgreichen Steuerung des Handelsgeschäfts“" (Schön 1997, S. 136) gesehen. Hauptzweck ist also die Eigeninformation des Kaufmanns (ebd., S. 138). Dies stimmt mit der österreichischen Sicht überein: ohne Wirtschaftsrechnung, zu der die externe Rechnungslegung gehört, gäbe es „,kein Mittel, zu erkennen, was rationell ist, und so könnte die Erzeugung bewußt nicht auf Wirtschaftlichkeit eingestellt werden“ (Mises 1920, S. 100). Die Rechnungslegung dient dazu, den Erfolg der wirtschaftlichen Tätigkeit zu messen (ebd., S. 112): Die „Buchführung, Bilanzaufstellung und Gewinn- und Verlustrechnung ermitteln die Ergebnisse des vollzogenen Handelns“ (Mises 1940, S. 198). Ob ein Unternehmen also am Markt positiv oder negativ sanktioniert wurde, erfährt es letztlich über seine Buchführung, indem mit deren Hilfe das Jahresergebnis - Gewinn oder Verlust - ermittelt wird.

Die (interne) Kosten- und Leistungsrechnung als weiterer Bestandteil der Wirtschaftsrechnung im Sinne von Mises gibt den Unternehmen Orientierung, um sich prospektiv für zukünftige Aufgaben zu positionieren. Diese Rechnungslegung, bei der interne Daten verwendet werden, die dem externen Informationsadressaten häufig verborgen bleiben, hilft den Unternehmern, unter den vielfältigen Einsatzmöglichkeiten für ihr Kapital eine ökonomisch sinnvolle Entscheidung zu treffen (Boettke 1998, S. 135), in Form der auf der Kosten- und Leistungsrechnung basierenden Kalkulation prägt sie die zukünftigen Handlungen von Unternehmen und Unternehmern (Mises 1940, S. 198), indem sie Prognosen ermöglicht.

Die Auswertung und Prognose der Marktpreise hilft somit Unternehmen und Unternehmern, sich mit ihren Handlungen am Markt zu orientieren, indem sie Gewinn- und Verlustschätzungen erlaubt. Die Orientierung und Prognose erfolgt dabei nicht nur an den eigenen Gewinnermittlungen, sondern auch anhand der Auswertungen und Prognosen anderer Unternehmer, indem die Rechnungslegung es ermöglicht, Bewertungen anderer ökonomischer Akteure zu beobachten (Huerta de Soto 2013, 118). Dabei ist es hilfreich, dass aufgrund der Rechnungslegung Vermögensgegenstände und Schulden monetär bewertet und so vergleichbar gemacht werden können, obwohl der Bewer- tungsprozess immer eine subjektive Beurteilung des Unternehmers ist. Rechnungslegungsvorschriften helfen aber, die stets subjektive Bewertung zu objektivieren. Ohne eine Wirtschaftsrechnung wäre das Wirtschaften als rationaler Umgang mit dem Phänomen der Knappheit in einer dynamischen und komplexen Welt nicht möglich. Sie bietet für die Unternehmen ,die einzige gesellschaftliche Orientierung, um Fehlanpassungen zu entdecken“ (Huerta de Soto 2013, 120).

\section{Bedeutung für die Weiterentwicklung der Rechnungslegung}

In der deutschen Rechnungslegungspraxis werden zwei Rechnungslegungssysteme genutzt: Die handelsrechtliche Rechnungslegung nach dem Handelsgesetzbuch (HGB) und die International Financial Reporting Standards (IFRS). Das Handelsgesetzbuch wurde durch das Bilanzrechtsmodernisierungsgesetz (BilMoG) im Jahre 2010 grundlegend reformiert und an die Entwicklungen der internationalen Rechnungslegung angepasst. Für die IFRS wird eine Weiterentwicklung laufend diskutiert.

\subsection{HGB-Rechnungslegung}

Wichtigster Zweck der HGB-Bilanzierung ist die Zahlungsbemessung, ergänzt durch die eine ,objektivierte Informationsvermittlung“ (Küting/Lam 2013, S. 1738) durch die Rechnungslegung. Eine besondere Bedeutung hat im Rahmen der Zahlungsbemessung das in $\S 252$ Abs. 1 Nr. 4 HGB festgeschriebene Vorsichtsprinzip. Mit der Bilanzierung nach dem Handelsrecht soll der Betrag ermittelt werden, der an die die Eigentümer des Unternehmens ausgeschüttet werden kann, ohne dass die (haftende) Substanz des Unternehmens unter einen Mindestbetrag sinkt. Ohne die Gewinnermittlung durch die Handelsbilanz ist nicht feststellbar, ob die entnommenen Beträge tatsächlich aus den erwirtschafteten Gewinnen des Unternehmens resultieren. Ohne eine Gewinnfeststellung ließe sich weder ex post der Unternehmenserfolg ermitteln, noch ex ante die Geschäftstätigkeit planen. Der Gläubigerschutz ist eine besondere Ausprägung der Gewinnausschüttungsfunktion, da die mögliche Ausschüttung unter Berücksichtigung der Ansprüche der Fremdkapitalgeber ermittelt wird.

Aus diesem Grunde basiert die Bewertung in einem HGBJahresabschluss insbesondere auf Vergangenheitswerten: $\S 253$ Abs. 1 Satz 1 HGB setzt als Bewertungsgrundlage und -obergrenze für Vermögensgegenstände die Anschaffungs- oder Herstellungskosten fest. Diese legen den objektivierenden Rahmen fest, innerhalb dessen sich das bilanzierende Unternehmen bei der immer subjektiven Bewertung seiner Vermögensgegenstände bewegen kann. Damit ist ein Mangel verbunden, der insbesondere die Informationsfunktion der Rechnungslegung betrifft. Es ist gut möglich, dass ein Vermögensgegenstand nach der Anschaffung oder Herstellung an Wert gewinnt. Diese Wertsteigerung wird nach HGB aber nicht abgebildet, da die Bewertung durch die Anschaffungs- oder Herstellungskos- 
ten nach oben gedeckelt ist. Die Information, dass Vermögensgegenstände im Wert über die Anschaffungs- oder Herstellungskosten gestiegen sind, wird in einem handelsrechtlichen Abschluss also bewusst verschwiegen. Die asymmetrische Informationsverteilung zwischen Rechnungslegenden und Adressaten der Rechnungslegung wird damit nicht so weit abgebaut, wie durch einen in diesem Sinne korrekten Ausweis der Wertsteigerung möglich ist. Das Handelsrecht geht mit dieser Selbstbeschränkung auf die Schwierigkeiten ein, die korrekte Wertsteigerung zu ermitteln, solange sie nicht durch eine Markttransaktion bestätigt wurde. Aus wohlfahrtsökonomischer Sicht verbleibt aber dieser Mangel.

Dagegen stehen die Vorteile dieser vorsichtigen Rechnungslegung: keine Kapitalaufzehrung und die Orientierung an Marktpreisen. Durch die Orientierung an Marktpreisen wird die marktwirtschaftliche Funktion der Preise (Anzeige von relativen Knappheiten aufgrund der Wissensmitteilung der Marktteilnehmer) in die Rechnungslegung transportiert. Somit laufen die bilanzierenden Unternehmen und die externen Bilanzadressaten nicht Gefahr, sich an hypothetischen Werten zu orientieren, in denen statt des Wissens der Marktteilnehmer lediglich die Erwartungen und Einschätzungen des Bilanzierenden eingehen.

\subsection{Internationale Rechnungslegung}

Die angelsächsisch geprägte internationale Rechnungslegung setzt im Gegensatz zur HGB-Rechnungslegung ausschließlich auf die Informationsfunktion der Rechnungslegung. Dabei geht sie - anders als das prinzipienorientierte HGB - weitgehend kasuistisch vor. Dies führt dazu, dass die IFRS ein außerordentlich umfangreiches Werk darstellen.

Mit Hilfe von IFRS-Jahresabschlüssen sollen „Abschlussinformationen zur Verfügung gestellt werden, die (potenziellen) Investoren und (potenziellen) Gläubigern nützlich für deren spezifische, mit dem Unternehmen verbundene Entscheidungsfindung sind“ (Küting/Lam 2013, S. 1741). Dies führt dazu, dass die zukünftigen Nettomittelzuflüsse des bilanzierenden Unternehmens im Fokus der Rechnungslegung stehen, so dass es zu einer weitreichenden Nutzung von Schätzungen, Ermessensentscheidungen und Modellen unter Verwendung von Annahmen im Rahmen der Bewertung kommt (ebd.). Beispiele sind der Fair Value sowie der Nutzungswert.

IFRS 13.9 definiert den Fair Value als den Preis, der bei der Veräußerung eines Vermögenswertes oder bei der Übertragung einer Verbindlichkeit im Rahmen einer gewöhnlichen Transaktion zwischen Marktteilnehmern am Bewertungsstichtag erhalten bzw. gezahlt würde. ,Somit basiert das Fair-Value-Konzept auf einer hypothetischen Transaktion“ (IDW 2014, 85), und nicht auf einer tatsächlich statt gefundenen Markttransaktion. Dies kommt auch darin zum Ausdruck, dass als Bewertungsverfahren nach IFRS $13.61 \mathrm{ff}$. nicht nur marktpreisorientierte Verfahren zur Ermittlung des Fair Value in Betracht kommen, sondern auch kapitalwert- oder kostenorientierte Verfahren.
Nach IAS 36 ist unter bestimmten Bedingungen ein Werthaltigkeitstest (Impairment test) zur Beurteilung der Wertminderung von Vermögenswerten vorzunehmen. Dabei wird der Buchwert eines Vermögenswertes mit dem erzielbaren Betrag verglichen. Der erzielbare Betrag ist der höhere Wert von Fair Value abzüglich der Veräußerungskosten und dem Nutzungswert. Der Nutzungswert wiederum ergibt sich aus den diskontierten (zukünftigen) NettoCashflows bei bestimmungsgemäßer Nutzung und dem Verkauf des Vermögenswertes.

Da sowohl der Fair Value als auch der Nutzungswert durch Zukunftsabschätzungen bestimmt werden (können), sind in der IFRS-Rechnungslegung in erheblichen Maße subjektive, kaum objektivierbare Erwartungen enthalten. Dies kann unter anderem dazu führen, dass in einem IFRS-Abschluss nicht realisierte Gewinne ausgewiesen werden. Das hohe Maß an Subjektivität der Abschlüsse nach IFRS ist ein Problem, das zu beachten ist. Die Möglichkeit, Bewertungen mit Hilfe von Schätzungen, Modellannahmen und weiteren Ermessensspielräumen zu beeinflussen, kann dazu führen, dass der Bilanzierende nicht nur externe Bilanzleser beeinflusst, sondern auch die Entscheider im eigenen Unternehmen und diesen somit die Orientierungshilfe Rechnungslegung im komplexen und dynamischen Marktprozess verloren geht.

Weitere Probleme resultieren aber aus dem Ausweis unrealisierter Gewinne, wenn diese (teilweise) ausgeschüttet werden. Zwar haben die IFRS keine direkte Zahlungsbemessungsfunktion an die Unternehmenseigner, in der Praxis dienen sie aber häufig der Festlegung von Boni und anderen erfolgsabhängigen Vergütungen, so dass sie indirekt doch der Zahlungsbemessung dienen. Unternehmen, die auf Basis von IFRS-Abschlüssen Auszahlungen vornehmen, bewegen sich aber auf dünnem Eis: die Zahlung erfolgsabhängiger Vergütungen ist häufig realisiert, ohne dass es im Endeffekt zu einer Realisierung der ausgewiesenen Gewinne kommt.

\section{Zusammenfassung}

Rechnungslegung und Jahresabschlusserstellung werden häufig mit der Informations- und der Zahlungsbemessungsfunktion in Verbindung gebracht. Hinter der Informationsfunktion steht die Überlegung, dass die Bücher von einer besser informierten Geschäftsleitung für schlechter informierte Adressaten geführt und veröffentlicht werden. Die Rechnungslegung erfolgt dann für Unternehmensfremde und ist insbesondere deshalb von Bedeutung, weil der Markt aufgrund von Informationsasymmetrien zu Fehlallokationen führt bzw. im Extremfall gar nicht funktioniert. Diese wohlfahrtsökonomische Sichtweise wird aber durch die österreichische Sichtweise erweitert, nach der Markt und Rechnungslegung sich ergänzen, indem der Markt die Informationen bereit stellt, die Unternehmen dann im Rahmen der Buchhaltung auswerten, um im Markt erfolgreich agieren zu können. Dies resultiert daraus, dass die Unternehmen sich zunächst selbst 
informieren müssen, da sie nur mit Hilfe der Rechnungslegung die in den Preisen enthaltenen Marktinformationen auswerten können. Die Österreichische Schule der Nationalökonomie sieht also die Rechnungslegung weniger als eine Reaktion auf Fehlfunktionen des Marktes sondern vielmehr als eine zentrale Voraussetzung für das Funktionieren des komplexen und dynamischen Marktgeschehens. Die Rechnungslegung erfolgt in diesem Sinne für das Unternehmen selbst, gleichwohl hat sie auch eine Informationsfunktion für andere Unternehmen, indem diese sich an der Rechnungslegung anderer Unternehmen orientieren können.

Für die unterschiedlichen Rechnungslegungssysteme folgt daraus, dass sie sich nicht nur am Marktversagen asymmetrische Informationsverteilung orientieren dürfen, sondern auch die Selbstinformationsbedürfnisse der Unternehmen im Auge haben müssen, da diese ebenso nicht vollständig informiert sind und sich erst Informationen beschaffen müssen. Unternehmen laufen Gefahr, sich weniger an Marktpreisen und damit am zentralen Informationssystem einer marktwirtschaftlichen Ordnung als an subjektiven Ermessensentscheidungen zu orientieren, wenn in der Rechnungslegung Wertmaßstäbe zur Anwendung kommen, welche vornehmlich im Rahmen kaum objektivierbarer Ermessensspielräume entstehen, wie das bei dem Fair Value der International Financial Reporting Standards der Fall ist. Das HGB hat den Vorteil, als Bewertungsgrundlage und -obergrenze lediglich marktpreisbasierte Kategorien (Anschaffungs- oder Herstellungskosten) heranzuziehen. Den Nachteil, dass damit Informationen über Wertsteigerungen über die Anschaffungs- oder Herstellungskosten hinaus verloren gehen, nimmt das HGB dabei in Kauf.

Insofern hat dieser Beitrag versucht, eine breitere ökonomische Perspektive auf die verschiedenen Rechnungslegungssysteme zu vermitteln. Nicht nur die schlechter informierte Marktseite hat Informationsdefizite, sondern auch die besser informierte Marktseite muss zunächst einmal zu ihren Informationen gelangen. Dabei ist zu beachten, dass Informationsasymmetrien gegebenenfalls vergröBert werden, wenn in die durch die Rechnungslegung vermittelten Informationen gegebenenfalls zu stark Ermes- sensentscheidungen einfließen. Dies sollte bei der Weiterentwicklung von Rechnungslegungssystemen beachtet werden.

\section{Literatur:}

Akerlof, G., The Market For „Lemons“: Quality Uncertainty and the Market Mechanism, in: The Quarterely Journal of Economics 3/1970, S. 488-500.

Boettke, P. J, Living Economics - Yesterday, Today and Tomorrow, The Independent Institute 2012.

Boettke, P. J., Economic Calculation: The Austrian Contribution to Political Economy, in: Advances in Austrian Economics 5/ 1998, S. 131-158.

Daumann, F./Drewes, M., Ansätze einer evolutorischen Sportökonomik am Beispiel des Wettbewerbs in professionellen Sportligen, in: Klein/Kurscheidt (Hrsg.): Neue Perspektiven ökonomischer Sportforschung, Schorndorf 2007, S. 69-93.

Hayek, F. A., Sozialistische Wirtschaftsrechnung III: Wiedereinführung des Wettbewerbs, in: Wissenschaft und Sozialismus, Tübingen: Mohr Siebeck 2004, S. 121-148.

Hayek, F. A., Zur Bewältigung von Unwissenheit, in: Die AnmaBung von Wissen (Hrsg. Kerber, W.), Tübingen: Mohr 1996, S. 307-316.

Huerta de Soto, J., Sozialismus, Wirtschaftsrechnung und unternehmerische Funktion, Stuttgart: Lucius \& Lucius 2013.

Institut der Wirtschaftsprüfer (IDW), IDW Stellungnahme zur Rechnungslegung: Einzelfragen zur Ermittlung des Fair Value nach IFRS 13, in: IDW Fachnachrichten 1/2014, S. 84-99.

Küting, K./Lam, S., Der Zukunftsbezug in der Rechnungslegung nach HGB und IFRS im Vergleich, in: Der Betrieb 32/2013, S. 1737-1745.

Küting. K/Lauer, P., Die Bedeutung des Anschaffungskostenprinzips und die Folgen seiner Durchbrechung, in: Der Betrieb 22/ 2013, S. 1185-1191.

Littkemann, J., Holtrup, M., Schulte, K., Buchführung, Wiesbaden: Gabler 2010.

Mises, L., Die Wirtschaftsrechnung im sozialistischen Gemeinwesen, in: Archiv für Sozialwissenschaft und Sozialpolitik 47 (1920), S. 86-121.

Mises, L., Die Gemeinwirtschaft: Untersuchungen über den Sozialismus, Jena 1922.

Mises, L., Nationalökonomie: Theorie des Handelns und Wirtschaftens, Genf 1940.

Schön, W., Entwicklung und Perspektive des Handelsbilanzrechts: vom ADHGB zum IASC, in: Zeitschrift für das gesamte Handelsrecht und Wirtschaftsrecht 161 (1997), S. 134-159.

Zimmermann, J., Ordnungspolitische Fragen zur Unternehmenspublizität nach der Enron-Insolvenz, in: Wirtschaftsdienst 2002/ 5, S. 303-312. 